\title{
Una propuesta didáctica para la enseñanza del concepto de derivada ${ }^{1}$
}

\section{A methodological approach to the teaching of the concept of derivative}

\section{A abordagem metodológica para o ensino do conceito de derivada}

Recibido: mayode 2013

Aceptado: agosto de 2013
Darío Álvarez Mejía ${ }^{2}$

Humberto Colorado Torres ${ }^{3}$

Liliana Patricia Ospina $\mathrm{M}^{4}$

\section{Resumen}

El propósito de la investigación ${ }^{5}$ fue determinar la diferencia en el aprendizaje significativo del concepto de derivada y reglas de derivación, en dos grupos de estudiantes de cálculo diferencial de la Universidad del Quindío, en uno utilizando la estrategia didáctica de enseñanza orientada desde conceptos previos, recorrido histórico, fases real, simbólica y conceptual y la resolución de problemas, y en el otro la estrategia didáctica tradicional, el tipo de investigación fue comparativa y correlacional. El diseño metodológico es cuasiexperimental. Se aplicó la prueba t-student para definir los resultados entre los grupos. Se llegó a la conclusión de que la estrategia didáctica propuesta en la investigación permitió que los estudiantes del grupo experimental comprendieran con mayor claridad las temáticas tratadas.

Palabras clave: Enseñanza; metodología de enseñanza; trabajo en el aula; didáctica; matemáticas escolares; cálculo; derivación; aprendizaje significativo.

\begin{abstract}
The purpose of the research 1 was to determine the difference in meaningful learning of the concept of derivative and derivation rules in two groups of differential calculus students at the University of Quindio, one using didactic teaching strategy oriented from previous concepts, travel historical phases-real, symbolic and conceptual and problem solving, and on the other the traditional teaching strategy, the type of research was comparative and correlational. The methodological design is quasi-experimental. The t-test was used to define student outcomes between the groups. It is concluded that the
\end{abstract}

1 Artíulo de Investigación

2 Universidad del Quindío. Contacto: dariome@uniquindio.edu.co

3 Universidad del Quindío. Contacto: colorado@uniquindio.edu.co

4 Universidad del Quindío Contacto: lpospina@uniquindio.edu.co

5 Grupo de Investigación en Educación Matemática GEMAUQ, Programa de Licenciatura en Matemáticas, Universidad del Quindío 
teaching strategy research proposal allowed the experimental group students understand more clearly the topics addressed.

Keywords: Teaching, teaching methodology, classroom work, teaching, school mathematics, calculation, derivation; meaningful learning.

\section{Resumo}

Objetivo da pesquisa 1 foi determinar a diferença na aprendizagem significativa do conceito de regras derivados e derivação em dois grupos de estudantes de Cálculo Diferencial da Universidade de Quindío, usando uma estratégia de ensino didático orientado a partir de conceitos anteriores, viagens histórico fases-real, simbólico e conceitual e resolução de problemas, e por outro, a estratégia de ensino tradicional, o tipo de pesquisa foi comparativa e correlacional. $\mathrm{O}$ desenho metodológico é quasi-experimental. O teste $t$ foi utilizado para definir os resultados dos alunos entre os grupos. Conclui-se que a proposta de pesquisa a estratégia de ensino permitiu que os estudantes do grupo experimental compreender mais claramente os temas abordados.

Palavras-chave: Palavras-chave: ensino, metodologia de ensino, o trabalho em sala de aula, ensino, matemática escolar, o cálculo, a derivação; aprendizagem significativa.

\section{Presentación del problema}

Las dificultades encontradas en el área de matemática de acuerdo a los resultados de las evaluaciones del Tercer estudio Internacional sobre Matemáticas y Ciencias TIMSS, Pruebas SABER PRO, Programme for International Student Assessment PISA, indican que los estudiantes no alcanzan los niveles esperados en el área, situación que exige un gran desafío para mejorar la calidad de la educación. Las matemáticas son señaladas por las autoridades educativas de nuestro país, como la principal asignatura que presenta obstáculos para que los estudiantes puedan seguir adelante en su formación académica. Cardona (2005) señala que "Históricamente las matemáticas han sido tema difícil pero importante dentro del currículo escolar y, tal vez por esta razón, se han utilizado como filtro para la educación superior". Según Moreno \& Ríos (2006) los docentes limitan su acción educativa a repetir los conceptos matemáticos tal como aparecen en los libros de texto o en la misma forma en que le fueron enseñados, reduciendo sus clases a una algoritmización de los conceptos del cálculo que los estudiantes contemplan, memorizan y repiten en los exámenes. Estas dificultades también se reflejan en los programas de la Universidad del Quindío, la falta de comprensión de los conceptos matemáticos limita los desempeños de los estudiantes en los cursos de matemáticas, conllevando al fracaso y la deserción universitaria, en especial los estudiantes que ven cálculo diferencial. (Sánchez, 2007). Artigue et al (1995) afirman que es una enseñanza marcada por la manipulación de fórmulas evidenciada en la determinación del límite, derivada o integral de una función, en lugar del análisis de estos conceptos y su aplicación en la solución de los problemas del entorno académico y social del estudiante. En consecuencia surge como pregunta de investigación:

¿Una estrategia didáctica de enseñanza orientada desde los conceptos previos, el recorrido histórico, las fases -real, simbólica y conceptual- y la resolución de problemas, promoverá el aprendizaje significativo del concepto de derivada y las reglas de derivación en los estudiantes de cálculo diferencial de la Universidad del Quindío? 


\section{Marco de referencia conceptual}

Para dar una mayor comprensión de lo anterior es importante reconocer algunos conceptos básicos y necesarios para la fundamentación teórica de esta propuesta de investigación, entre los cuales están: aprendizaje significativo, importancia de la teoría del aprendizaje significativo en la enseñanza de las matemáticas, cálculo diferencial, la estrategia didáctica tradicional, las etapas de la estrategia didáctica de enseñanza orientada desde los conceptos previos, el recorrido histórico, fase-real, simbólica y conceptualy la resolución de problemas.

Aprendizaje Significativo. Un aprendizaje es significativo cuando los contenidos son relacionados de modo no arbitrario y sustancial (no al pie de la letra) con lo que el estudiante ya sabe. Esto quiere decir, que en el proceso educativo es importante considerar lo que el individuo ya sabe de tal manera que establezca una relación con aquello que debe aprender. Este proceso tiene lugar si el educando tiene en su estructura cognitiva conceptos, estos son: ideas, proposiciones claras, con los cuales la nueva información puede interactuar. Ausubel (1983:18).

Importancia de la teoría del aprendizaje significativo en la enseñanza de las matemáticas. Este tipo de aprendizaje pretende buscar que el estudiante construya su propio aprendizaje, llevándolo hacia la autonomía al momento de pensar, de modo tal que desarrolle su inteligencia relacionando de manera integral lo que tiene y conoce respecto a lo que se quiere aprender... que integren sus experiencias a otras ya conocidas, que elijan lo que desean aprender y no buscar el desarrollo de la memoria y la repetición como alternativa de aprendizaje. Este tipo de aprendizaje modifica la estructura cognitiva del estudiante mediante reacomodos de la misma para integrar a la nueva información; además, puede adquirir nuevos conocimientos de la matemática con mayor facilidad, relacionando los ya aprendidos con los nuevos en forma significativa (Murillo, 2000).

\section{Metodología}

Se realizó una investigación de tipo comparativa y correlacional. La población objeto de estudio fue definida por todos los grupos de estudiantes que cursan la asignatura de cálculo diferencial de la Universidad del Quindío. La muestra corresponde a dos grupos: control y experimental. Se tuvo en cuenta que los grupos que ingresaran cumplieran con las condiciones de homogeneidad donde los estudiantes presentaran las mismas características en términos de los presupuestos teóricos, dadas las condiciones de oportunidad, tiempo, lugar y disponibilidad de los investigadores se seleccionaron los grupos experimental y control del Programa de Topografía. El diseño metodológico es cuasiexperimental.

Las fases que se desarrollaron fueron: a) Se definieron los grupos experimental y control, b) Se elaboró y desarrolló la estrategia didáctica tradicional del concepto de derivada y las reglas de derivación en el grupo control, c) Se elaboró y desarrolló la estrategia didáctica orientada desde los conceptos previos, el recorrido histórico, las fases-real, simbólica y conceptual- y la resolución de problemas en el grupo experimental, d) Se elaboró y aplicó el postest a ambos grupos con el fin de reconocer si los estudiantes adquirieron aprendizajes significativos, e) Procesamiento de la información: se tabuló la base de datos del pre-test y del postest. Para cada grupo se presentaron los resultados en términos de frecuencia y porcentajes, en cuadros y gráficas estadísticas. Se aplicó la prueba t-student para definir los resultados entre los grupos comparados, f) Se realizó el análisis y la interpretación de los resultados, con el fin de contrastar los fundamentos teóricos con los resultados.

Gráfico de cajas 1: Resultados pretest grupos control y experimental

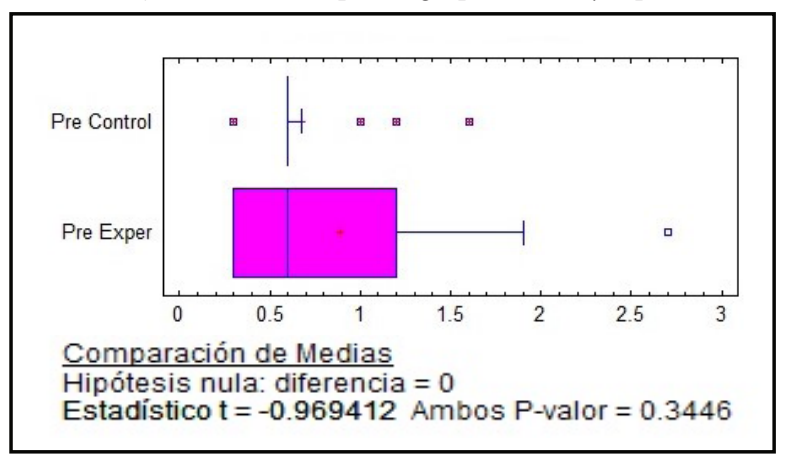

Fuente: Elaboración propia

\section{Análisis de datos}

Las siguientes graficas muestran los resultados obtenidos por los grupos control y experimental. 
Como los resultados de $\mathrm{P}$ en la prueba $\mathrm{t}$, son superiores al $=0.05$ (nivel de significancia) entonces se afirma que no existen diferencias significativas entre los grupos control y experimental en el momento de aplicar el pretest, entonces se concluye que ambos grupos reunen las condiciones de homogeneidad que se requieren para la aplicación de los modelos a comparar.

Gráfico de cajas 2: Resultados postest grupos control y experimental

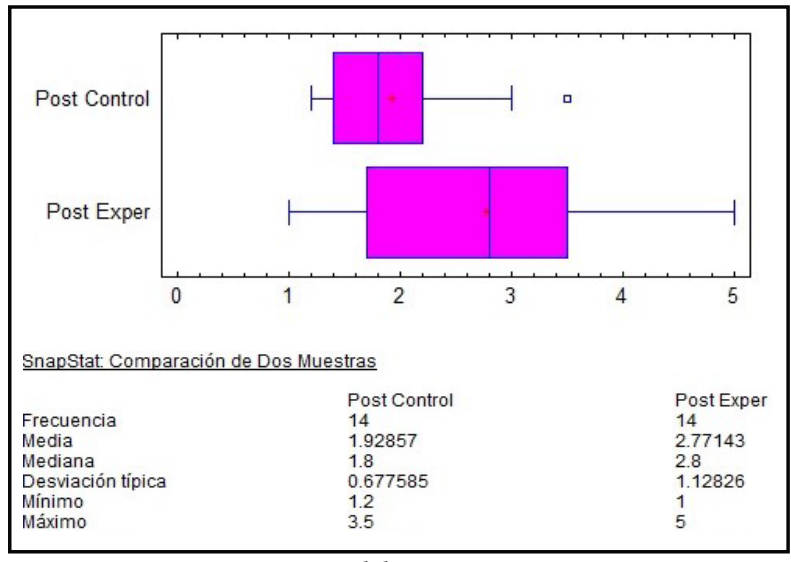

Fuente: Elaboración propia

Los resultados obtenidos en $\mathrm{P}$, en la prueba $\mathrm{t}$ son inferiores $\mathrm{al}=0.05$ (nivel de significancia) entonces se afirma que existen diferencias significativas entre los grupos control y experimental de acuerdo con los resultados obtenidos al aplicar el postest. Lo que indica que la estrategia didáctica aplicada al grupo experimental tuvo mayor incidencia significativa en el proceso de aprendizaje.

Gráfico de cajas 3: Resultados pretest y postest grupo experimenta

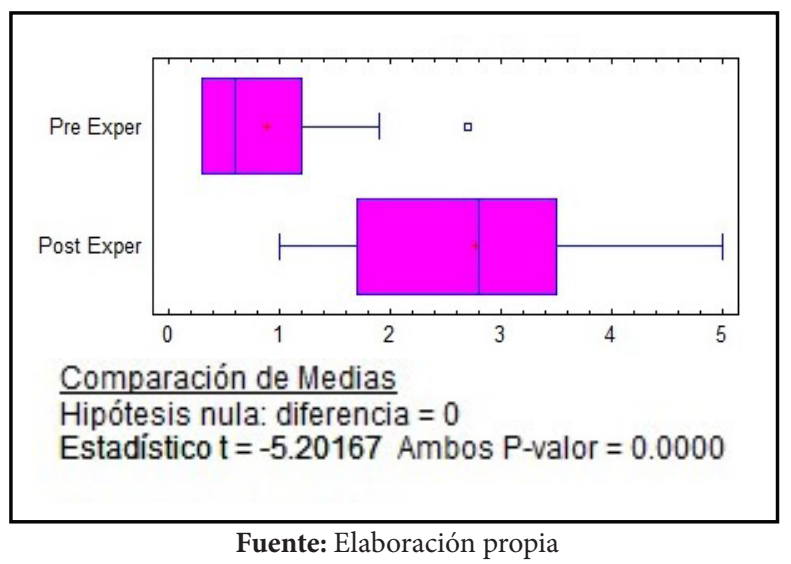

Los resultados obtenidos en $\mathrm{P}$ en la prueba $\mathrm{t}$ son inferiores $\mathrm{al}=0.05$ (nivel de significación), entonces se afirma que existe diferencias significativas entre los resultados del pretest y del postest aplicados al grupo experimental.

Gráfico de cajas 4: Resultados pretest y postest grupo control

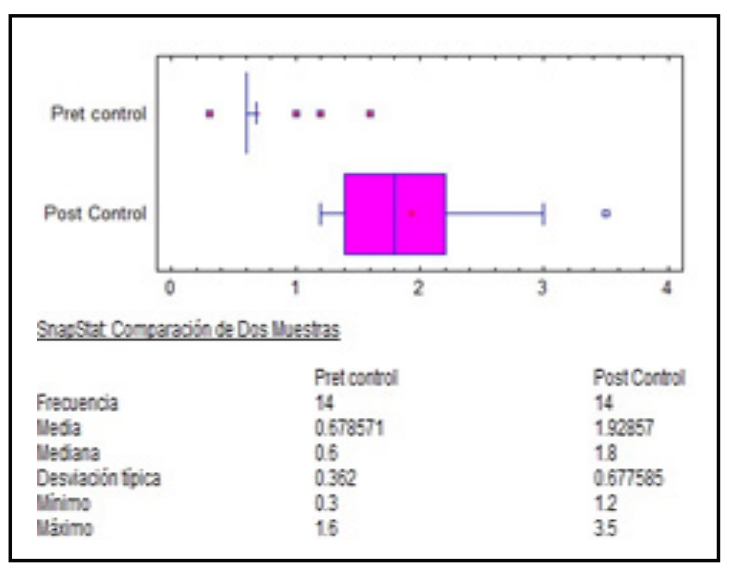

Fuente: Elaboración propia

Los resultados obtenidos en $\mathrm{P}$ en la prueba $\mathrm{t}$ son inferiores al $=0.05$ (nivel de significación), entonces se afirma que existe diferencias significativas entre los resultados del pretest y del postest aplicados al grupo control.

\section{Discusión}

Para el abordaje de la propuesta, la hipótesis de investigación tuvo en cuenta la estrategia didáctica orientada desde los conceptos previos, el recorrido histórico, las fases- real, simbólica y conceptual y la resolución de problemas; de esta manera se inició con algunos conocimientos previos que sustentan el concepto de la derivada y las reglas de derivación, tales como: función, pendiente, tangente, límite, continuidad, infinito, velocidad, etc. Por tal razón Murillo (2000), plantea que para alcanzar un aprendizaje significativo en una clase de matemática se deben tener presentes las experiencias y conocimientos previos de los estudiantes, los cuales son punto de partida para el proceso de enseñanza y se deben preparar los contenidos de acuerdo con la etapa de razonamiento por la que atraviese el estudiante, pues no se pretende que construya un aprendizaje si previamente no ha adquirido conocimientos previos del tema para relacionarlos con los nuevos. 
Otro elemento fundante en la conceptualización de la derivada y las reglas de derivación que se tuvo en cuenta fue: la dimensión Histórico-Epistemológica, que centra el estudio historiográfico del concepto de derivada con el fin de identificar los problemas que hicieron necesario el concepto de ella, las soluciones que se alcanzan en ciertas épocas, las lagunas y los obstáculos epistemológicos que detuvieron o impidieron el desarrollo de este concepto durante ciertos períodos de la historia. Godino (2004) afirma que es muy útil conocer la génesis histórica de los contenidos que se quieren enseñar, ya que ésta puede ser una fuente importante de material para su enseñanza. Considerar el momento histórico en el que se desarrolla un contenido matemático lleva a hablar de sus conexiones con la ciencia de la época, con las necesidades humanas, sociales o de cualquier otro tipo que llevaron al inicio y posterior desarrollo de dicho contenido. También obliga a hablar de las aplicaciones posteriores, esperadas o que surgieron de forma imprevista. Otro elemento a destacar es que la historia también puede ayudar a resolver el problema de la motivación del estudiante.

Una de las etapas de la estrategia didáctica fue la fase Real con la cual se busca que el estudiante visualice el concepto de la derivada y las reglas de derivación en situaciones reales a través de representaciones con videos, gráficas, fotografías y dibujos con el fin de que realice conjeturas, justifique su pensamiento matemático, reconozca y describa patrones, realice analogías para que empiece a construir los conceptos. Los estudiantes necesitan ver las conexiones entre conceptos y sus aplicaciones. A medida que relacionan ideas matemáticas con experiencias cotidianas y situaciones del mundo real, se van dando cuenta que esas ideas son útiles y poderosas. El conocimiento matemático de los estudiantes aumenta a medida que entienden que varias representaciones se interrelacionan (ej: física, verbal, numérica, y gráfica). Para lograrlo necesitan experimentar con cada una y entender cómo están conectadas (Vasco, 1994).

Una vez los estudiantes son conscientes de las características y propiedades aprendidas en la fase anterior, se les muestra la forma de representarlo con símbolos matemáticos (fase simbólica). Existen representaciones mentales, conjunto de imágenes, conceptos, nociones, ideas, creencias, concepciones que un individuo puede tener sobre un objeto, sobre una situación y sobre aquello que les está asociado. "Permiten una mirada del objeto en ausencia total de significante perceptible". Las representaciones mentales están ligadas a la interiorización de representaciones externas, de la misma manera que las imágenes mentales lo están a una interiorización de los preceptos (Godino, 2004).

Las anteriores fases son elementos constitutivos para el trabajo en la fase conceptual, la cual tiene como objetivo establecer y completar la red de relaciones del concepto de derivada y las reglas de derivación, relacionar los contenidos, conectar tópicos del mismo campo conceptual, deducir conclusiones lógicas, razonar inductiva y deductivamente, realizar demostraciones, realizar la modelación y la ejercitación de los conceptos. En esta fase se muestra un resumen de todo lo aprendido a través de mapas conceptuales o redes semánticas, los cuáles son representaciones gráficas de los esquemas de conocimiento; que permiten que el estudiante piense, construya y haga elaboraciones mentales de los conceptos.

Finalmente se trabajó la fase de resolución de problemas donde se les propone a los estudiantes situaciones con el fin de apliquen los conceptos adquiridos. La actividad de resolver problemas es esencial si quiere conseguir un aprendizaje significativo de las matemáticas. No se debe pensar en esta actividad sólo como un contenido más del currículo matemático, sino como uno de los vehículos principales del aprendizaje de conceptos y habilidades matemáticas, y una fuente de motivación para los estudiantes ya que permite contextualizar y personalizar los conocimientos. Al resolver un problema, el estudiante dota de significado las prácticas matemáticas realizadas, ya que comprende su finalidad (Godino, 2004).

Con la estrategia didáctica de enseñanza del concepto de derivada y las reglas de derivación, orientada desde los conceptos previos, el recorrido histórico, las fases: real, simbólica y conceptual y la resolución de problemas, se logró despertar en los estudiantes el interés por los temas abordados, mostrando la pertinencia y aplicación de los conceptos en el contexto, logrando además, que los estudiantes progresarán en su forma de razonar y analizar el concepto de la derivada y las reglas de derivación, conduciendo así 
a que el aprendizaje sea significativo y no por mera repetición.

En el grupo control se orientó el concepto de la derivada y las reglas de derivación utilizando la estrategia didáctica tradicional. La concepción tradicional de la enseñanza del cálculo, se limita al desarrollo de unas clases que se reducen a exposiciones de conceptos planteados en situaciones problemáticas que se ilustran con ejercicios o problemas descontextualizados, donde el énfasis se coloca en la memorización de técnicas y reglas que no tienen vinculación con la realidad (Vera y Silva, 2005).

Teniendo en cuenta lo anterior, en el grupo control el concepto de la derivada y las reglas de derivación se orientó dando la definición, la interpretación geométrica y física, se explicaron algunos ejemplos, se dieron a conocer las reglas de derivación y por último se desarrollaron problemas de aplicación; es importante resaltar que además de lo anterior se realizaron actividades extra clase como: ejercicios y talleres, con el fin de que los estudiantes profundizaran el concepto. Se observó que los estudiantes tuvieron algunos avances con respecto a su desempeño que se evidenció en los resultados del pretest y postest, queriendo decir que con el modelo tradicional los estudiantes también lograron avanzar en su aprendizaje.

\section{Conclusiones}

- A través de la aplicación de la estrategia didáctica orientada desde los conceptos previos, el recorrido histórico, las fases -real, simbólica y conceptual- y la resolución de problemas, se logró que los estudiantes del grupo experimental adquirieran un aprendizaje significativo del concepto de la derivada y las reglas de derivación, evidenciado en la resolución de problemas de aplicación.

- Al comparar los resultados de los postest de ambos grupos, utilizando la prueba t-student, se evidenció que hay diferencias significativas, lo que indica que se acepta la hipótesis de trabajo y se rechaza la hipótesis nula, mostrándose así la eficacia de la estrategia didáctica orientada desde los conceptos previos, el recorrido histórico, las fases -real, simbólica y conceptual- y la resolución de problemas, alcanzando así el objetivo de la investigación.

- Se logró despertar interés y motivación de los estudiantes del grupo experimental hacia el tema, a través de las actividades didácticas como: la proyección de videos y la representación gráfica basados en situaciones reales.

\section{Referencias}

Álvarez, D., Barrantes, C. J., \& Ospina, L.P. (2005). Cómo lograr un aprendizaje significativo en la enseñanza de la función cuadrática. Cali: Universidad de San Buenaventura.

Álvarez, D., Colorado, H., \& Ospina, L.P. (2010). Didáctica de las Matemáticas-Una Experiencia Pedagógica Moderna. Armenia: Ediciones Elizcom.

Ausubel, N. (1983). Psicología educativa: Un punto de vista cognoscitivo. México: Trillas $2^{\circ}$ ed.

Artigue, M. et-al. (1995). Ingeniería didáctica en e ducación matemática. Un esquema para la investigación y la innovación en la enseñanza y el aprendizaje de las matemáticas. México: Grupo Editorial Iberoamericana.

Badillo, E. (2003). La derivada como objeto matemático y como objeto de enseñanza y aprendizaje en docentes de matemática de Colombia: la derivada un concepto a caballo entre la matemática y la física. (Tesis doctoral). Universidad Autónoma, Barcelona.

Cardona, E. (2005). La derivada de caratheadory" elementos para una transposición didáctica. Sexto Encuentro Colombiano y primero Iberoamericano de la enseñanza del Cálculo. (Tesis de Maestría). Pontificia Universidad Javeriana, Cali.

Contreras, A. (2001). Una perspectiva de la enseñan za-aprendizaje de la continuidad y la derivada de una función en Bachillerato y Universidad. Revista de educación, ISSN 0034-8082, No 331, 2003 (Ejemplar dedicado a: La formación del docente universitario), pags. 399-419. Universidad de la Rioja. 
Díaz, F. \& Hernández, G. (1998). Estrategias docentes para un Aprendizaje Significativo, Una interpretación constructivista. México: Editorial Mcgraw Hill.

Flórez, R. (2000). Docente del Siglo XXI, cómo desarrollar una práctica docente competitiva, evaluación pedagogía y cognición. Colombia: Editorial Mcgraw Hill.

Godino, J et al. (2004). Didáctica de las matemáticas para docentes. España: Universidad de Granada.

Hernández S, R. et-al (1999). Metodología de la Investigación. México: Editorial McGraw-Hill.
Moreno, C. \& Rios, P. (2006). Concepciones en la enseñanza del cálculo. SAPIENS, volumen (7), no.2, pp. 25-39.

Murillo, P. (2004). El aprendizaje significativo y la motivación. Recuperado de http://www.espaciopedagogico.com.

Vasco, C. (1994). Un nuevo enfoque para la didáctica de las matemáticas. Volumen I y II, Bogotá: Serie Pedagogía y Currículo, Ministerio de Educación Nacional.

Vera, H \& Silva, M. (2005.). Una propuesta educativa en informática educacional para la enseñanza de la matemática. Recuperado de http://www. sadpro.ucv.ve/agenda/online/vo16n17a0.html. 